\title{
QCD evolution equations from conformal symmetry
}

\author{
Vladimir Braun \\ Regensburg University, D-93040, Regensburg, Germany \\ E-mail: vladimir.braunephysik.uni-regensburg.de
}

\section{Alexander Manashov ${ }^{* \dagger}$}

Regensburg University, D-93040, Regensburg, Germany \& St.-Petersburg University, 199034,

St.-Petersburg, Russia

E-mail: alexander.manashov@physik.uni-regensburg.de

\begin{abstract}
QCD evolution equations in MS-like schemes can be recovered from the same equations in a modified theory, QCD in non-integer $d=4-2 \varepsilon$ dimensions, which enjoys exact scale and conformal invariance at the critical point. Restrictions imposed by the conformal symmetry of the modified theory allow one to obtain complete evolution kernels in integer (physical) dimensions at the given order of perturbation theory from the spectrum of anomalous dimensions added by the calculation of the special conformal anomaly at one order less. We use this technique to derive two-loop evolution equations for flavor-nonsinglet quark-antiquark light-ray operators that encode the scale dependence of generalized hadron parton distributions.
\end{abstract}

Loops and Legs in Quantum Field Theory - LL 2014,

27 April - 2 May 2014

Weimar, Germany

\footnotetext{
* Speaker.

${ }^{\dagger}$ The work by A.M. was supported by the DFG, grant BR2021/5-2.
} 


\section{Introduction}

Studies of hard exclusive reactions constitute an important part of the research programs at all major accelerator facilities. The theoretical description of such processes involves operator matrix elements between states with different momenta, dubbed generalized parton distributions (GPDs), or vacuum-to-hadron matrix elements, the distribution amplitudes (DAs). Scale dependence of these distributions is governed by the renormalization group (RG) equations for the corresponding (nonlocal) operators and are known, at present, to the two-loop accuracy [1-3]. This is one order less compared to the RG equations for the corresponding "inclusive" distributions involving forward matrix elements $[4,5]$ and closing this gap is desirable. The direct calculation is very challenging. Moreover, since the two-loop RGEs for GPDs are already very cumbersome, finding a suitable representation for the results becomes part of the problem.

It has been known for a long time [6] that one-loop evolution kernels can be restored from the corresponding anomalous dimensions thanks to conformal symmetry of the QCD Lagrangian. The generalization of this technique beyond the leading order was developed by D. Müller [7], who has shown that restrictions based on conformal symmetry allow one to restore full evolution kernels at given order of perturbation theory from the spectrum of anomalous dimensions at the same order, and the calculation of the special conformal anomaly at one order less. This technique was used to calculate the two-loop evolution kernels in momentum space for the GPDs [1-3, 8-10]. In Refs. $[11,12]$ we suggested a different approach to achieve the same goal. Instead of studying effects of the conformal symmetry breaking in the physical theory [8-10] it was proposed to make use of the exact conformal symmetry of a modified theory - QCD in $d=4-2 \varepsilon$ dimensions at critical coupling. Exact conformal symmetry simplifies considerably the analysis and also suggests the optimal representation for the results in terms of light-ray operators. We expect that this technique will become increasingly advantageous in higher orders.

This approach was illustrated in [11] on several examples to the two- and three-loop accuracy for scalar theories and used in [12] to obtain two-loop evolution equations for the flavor flavornonsinglet light-ray operators. The applications to gauge theories involve several subtleties that will be discussed below.

\section{Preliminaries}

Conformal symmetry transformations have the simplest form for the so-called light-ray operators that can be understood as generating functions for the renormalized leading-twist local operators:

$$
[\mathscr{O}]\left(x ; z_{1}, z_{2}\right) \equiv\left[\bar{q}\left(x+z_{1} n\right) \not q q\left(x+z_{2} n\right)\right] \equiv \sum_{m, k} \frac{z_{1}^{m} z_{2}^{k}}{m ! k !}\left[\left(D_{n}^{m} \bar{q}\right)(x) \not\left(D_{n}^{k} q\right)(x)\right] .
$$

Here $q(x)$ is a quark field, the Wilson line is implied between the quark fields on the light-cone, $D_{n}=n_{\mu} D^{\mu}$ is a covariant derivative, $n^{\mu}$ is an auxiliary light-like vector, $n^{2}=0$, that ensures symmetrization and subtraction of traces of local operators. The square brackets [...] stand for the renormalization in the MS scheme. We will tacitly assume that the quark and antiquark have different flavor so that there is no mixing with gluon operators. In most situations the overall coordinate $x$ is irrelevant and can be put to zero; we will often abbreviate $\mathscr{O}\left(z_{1}, z_{2}\right) \equiv \mathscr{O}\left(0 ; z_{1}, z_{2}\right)$. 
The RGE fot light-ray operators takes the form [14] (here and below $a=\alpha_{s} / 4 \pi$ )

$$
\left(M \partial_{M}+\beta(a) \partial_{a}+\mathbb{H}(a)\right)\left[\mathscr{O}\left(z_{1}, z_{2}\right)\right]=0,
$$

where $\mathbb{H}$ is an integral operator acting on the quark light-cone coordinates, $z_{i}$. It can be written as

$$
\mathbb{H}[\mathscr{O}]\left(z_{1}, z_{2}\right)=\int_{0}^{1} d \alpha \int_{0}^{1} d \beta h(\alpha, \beta)[\mathscr{O}]\left(z_{12}^{\alpha}, z_{21}^{\beta}\right),
$$

where $z_{12}^{\alpha} \equiv z_{1} \bar{\alpha}+z_{2} \alpha, \bar{\alpha}=1-\alpha$ and

$$
h(\alpha, \beta)=a h^{(1)}(\alpha, \beta)+a^{2} h^{(2)}(\alpha, \beta)+\ldots
$$

is a certain weight function (kernel). One can show that the powers $[\mathscr{O}]\left(z_{1}, z_{2}\right) \mapsto\left(z_{1}-z_{2}\right)^{N-1}$ are eigenfunctions of the operator $\mathbb{H}$, and the corresponding eigenvalues

$$
\gamma_{N}=\int d \alpha d \beta h(\alpha, \beta)(1-\alpha-\beta)^{N-1}
$$

are nothing else as the anomalous dimensions of local operators of spin $N$ [11]. The kernel $h(\alpha, \beta)$ is a function of two variables so that the knowledge of the anomalous dimensions $\gamma_{N}$ is not sufficient, in general, to find it. In a conformal theory, however, it is expected that the operator $\mathbb{H}$ commutes with the generators of the $S L(2)$ transformations, $\left[\mathbb{H}, S_{\alpha}\right]=0$. At the leading order the generators take the canonical form

$$
S_{+}^{(0)}=z_{1}^{2} \partial_{z_{1}}+z_{2}^{2} \partial_{z_{2}}+2\left(z_{1}+z_{2}\right), \quad S_{0}^{(0)}=z_{1} \partial_{z_{1}}+z_{2} \partial_{z_{2}}+2, \quad S_{-}^{(0)}=-\partial_{z_{1}}-\partial_{z_{2}} .
$$

Up to the trivial case $h(\alpha, \beta)=\delta(\alpha) \delta(\beta)$ the kernel of an operator commuting with the canonical generators (2.6) is a function of one variable only, $h(\alpha, \beta)=\bar{h}(\tau)$, where $\tau=\alpha \beta / \bar{\alpha} \bar{\beta}$ is the so-called conformal ratio. The function of one variable $\bar{h}(\tau)$ is determined uniquely by its moments (2.5) and can easily be reconstructed. It turns out that the one-loop kernel $h^{(1)}(\alpha, \beta)$ takes a remarkably simple form [15]

$$
h^{(1)}(\alpha, \beta)=-4 C_{F}\left[\delta_{+}(\tau)+\theta(1-\tau)-\frac{1}{2} \delta(\alpha) \delta(\beta)\right],
$$

where the regularized $\delta$-function, $\delta_{+}(\tau)$, is defined as

$$
\int d \alpha d \beta \delta_{+}(\tau) f\left(z_{12}^{\alpha}, z_{21}^{\beta}\right) \equiv \int_{0}^{1} d \alpha \int_{0}^{1} d \beta \delta(\tau)\left[f\left(z_{12}^{\alpha}, z_{21}^{\beta}\right)-f\left(z_{1}, z_{2}\right)\right] .
$$

Beyond one loop, conformal symmetry in QCD is broken by quantum corrections but, nevertheless, still imposes nontrivial constraints. We will show that: first, it is possible to construct the operators $S_{\alpha}(a)$ that commute with the evolution kernel $\mathbb{H}(a)$ in the four-dimensional interacting theory, $\left[\mathbb{H}(a), S_{\alpha}(a)\right]=0$ and, second, that this property guarantees that the kernel $\mathbb{H}(a)$ can be restored from its spectrum. To this end we will go over to the theory in noninteger, $d=4-2 \varepsilon$, dimensions at the intermediate steps. 


\section{QCD in $d=4-2 \varepsilon$ dimensions}

The QCD Lagrangian in $d=4-2 \varepsilon$ dimensional Euclidean space in covariant gauge has the form

$$
\mathscr{L}=\bar{q}(\not \partial-i g \not) q+\frac{1}{4} F_{\mu v}^{a} F^{a, \mu v}+\partial_{\mu} \bar{c}^{a}\left(D^{\mu} c\right)^{a}+\frac{1}{2 \xi}\left(\partial A^{a}\right)^{2} .
$$

For large number of flavours, $n_{f}$, the beta function

$$
\beta(a)=M \partial_{M} a=2 a\left(-\varepsilon-b_{0} a+\mathscr{O}\left(a^{2}\right)\right), \quad b_{0}=\frac{11}{3} N_{c}-\frac{2}{3} n_{f},
$$

has a nontrivial zero for the finely-tuned (critical) value of the coupling $a_{*}=-\varepsilon / b_{0}+O\left(\varepsilon^{2}\right)$. The theory thus enjoys exact scale and conformal invariance ${ }^{1}$ at the critical point $[16,17]$. As a consequence, the RGEs are exactly conformally invariant, but the generators are modified by quantum corrections as compared to their canonical expressions (2.6):

$$
S_{\alpha}=S_{\alpha}^{(0)}+a_{*} \Delta S_{\alpha}^{(1)}+a_{*}^{2} \Delta S_{\alpha}^{(2)}+\ldots
$$

One can show that the generator $S_{-}$(translation) does not receive any corrections, $S_{-}=S_{-}^{(0)}$, the deformation of $S_{0}$ can be calculated exactly in terms of the evolution operator (to all orders in perturbation theory) [11], whereas the deformation of $S_{+}$is nontrivial and has to be calculated explicitly to the required accuracy [12]:

$$
\begin{aligned}
& S_{0}=S_{0}^{(0)}-\varepsilon+\frac{1}{2} \mathbb{H}\left(a_{*}\right), \quad \mathbb{H}\left(a_{*}\right)=a_{*} \mathbb{H}^{(1)}+a_{*}^{2} \mathbb{H}^{(2)}+\ldots \\
& S_{+}=S_{+}^{(0)}+\left(z_{1}+z_{2}\right)\left(-\varepsilon+\frac{1}{2} a_{*} \mathbb{H}^{(1)}\right)+a_{*}\left(z_{1}-z_{2}\right) \Delta_{+}+\mathscr{O}\left(\varepsilon^{2}\right),
\end{aligned}
$$

where

$$
\Delta_{+}[\mathscr{O}]\left(z_{1}, z_{2}\right)=-2 C_{F} \int_{0}^{1} d \alpha\left(\frac{\bar{\alpha}}{\alpha}+\ln \alpha\right)\left[[\mathscr{O}]\left(z_{12}^{\alpha}, z_{2}\right)-[\mathscr{O}]\left(z_{1}, z_{21}^{\alpha}\right)\right]
$$

From the technical point of view this calculation replaces evaluation of the conformal anomaly in the theory in integer dimensions in the approach due to D. Müller [7].

The evolution kernel at the critical point has to commute with the symmetry generators, $\left[S_{\alpha}\left(a_{*}\right), \mathbb{H}\left(a_{*}\right)\right]=0$. Taking into account Eq. (3.4) one concludes that $\mathbb{H}\left(a_{*}\right)$ commutes with the two canonical generators, $\left[S_{-}^{(0)}, \mathbb{H}\left(a_{*}\right)\right]=\left[S_{0}^{(0)}, \mathbb{H}\left(a_{*}\right)\right]=0$, while expanding the last commutator in series in $a_{*}$ one obtains a nested set of commutator relations [11]

$$
\left[S_{+}^{(0)}, \mathbb{H}^{(1)}\right]=0, \quad\left[S_{+}^{(0)}, \mathbb{H}^{(2)}\right]=\left[\mathbb{H}^{(1)}, \Delta S_{+}^{(1)}\right], \quad\left[S_{+}^{(0)}, \mathbb{H}^{(3)}\right]=\left[\mathbb{H}^{(1)}, \Delta S_{+}^{(2)}\right]+\left[\mathbb{H}^{(2)}, \Delta S_{+}^{(1)}\right]
$$

etc. Note that the commutator of the canonical generator $S_{+}^{(0)}$ with the evolution kernel $\mathbb{H}^{(k)}$ on the 1.h.s. is given in terms of the kernels $\mathbb{H}^{(m)}$ and the corrections to the generator $\Delta S_{+}^{(m)}$ of order, $m<k$. The relations (3.7) can be viewed as inhomogeneous first-order differential equations on the kernels $\mathbb{H}^{(k)}$. Their solution determines $\mathbb{H}^{(k)}$ up to an $S L(2)$-invariant term (solution of the

\footnotetext{
${ }^{1}$ QCD is critically equivalent to the Non-Abelian Thirring model [17] that allows one to develop technique for calculation critical indices different from the standard perturbative expansion, see e.g. Refs. [18-21]
} 
corresponding homogeneous equation $\left[\mathbb{H}_{i n v}^{(k)}, S_{\alpha}^{(0)}\right]=0$ ), which can be restored from the spectrum of the anomalous dimensions. This procedure is described in detail in Ref. [11].

Last but not least, it is well known that in MS-like schemes the evolution kernels (anomalous dimensions) do not depend on the space-time dimension. It means that the kernel $\mathbb{H}(a)$ can be restored from the kernel at the critical point, $\mathbb{H}\left(a_{*}\right)$, simply by replacing $a_{*} \rightarrow a$ in the power series for $\mathbb{H}\left(a_{*}\right)$. Finally, rewriting $\varepsilon$ in terms of the critical coupling, $\varepsilon=-b_{0} a_{s}^{*}+\mathscr{O}\left(a_{s}^{* 2}\right)$, in the generators $S_{0}\left(a_{*}\right), S_{+}\left(a_{*}\right)$ one immediately concludes that the generators $S_{\alpha}(a)$, commute with the kernel $\mathbb{H}(a)$. In this way the evolution kernel in four-dimensional theory inherits the symmetries of the evolution kernel in conformal theory.

\section{Conformal Ward Identities}

To begin with, action of the generators $S_{\alpha}$ on the light-ray operator (which is auxiliary and scheme-dependent object) has to be defined in a consistent way. We will do this by expanding the light-ray operator over local conformal operators that can be classified according to their transformation properties with respect to the conformal group. These are determined by the nature of the critical point and are scheme-independent (i.e. can be viewed as "physical" observables).

The transformation laws for the leading-twist operators are completely fixed by their critical dimension and spin. A local operator that transforms under dilatation (D) and special conformal transformation $\left(\mathbf{K}^{\mu}\right)$ as follows

$$
\begin{aligned}
i\left[\mathbf{D},\left[\mathscr{O}_{N}\right](x)\right] & =\left(x \partial_{x}+\Delta_{N}^{*}\right)\left[\mathscr{O}_{N}\right](x), \\
i\left[\mathbf{K}^{\mu},\left[\mathscr{O}_{N}\right](x)\right] & =\left[2 x^{\mu}(x \partial)-x^{2} \partial^{\mu}+2 \Delta_{N}^{*} x^{\mu}+2 x^{v}\left(n^{\mu} \frac{\partial}{\partial n^{v}}-n_{v} \frac{\partial}{\partial n_{\mu}}\right)\right]\left[\mathscr{O}_{N}\right](x) .
\end{aligned}
$$

is called a conformal operator, by definition. The light-ray operator can be expanded over the basis of conformal operators $\mathscr{O}_{N k}(x)=\partial_{+}^{k}\left[\mathscr{O}_{N}\right](x)$ where $\partial_{+} \equiv(n \partial)$ with certain coefficient functions

$$
\left[\mathscr{O}\left(x ; z_{1}, z_{2}\right)\right]=\sum_{N k} \Psi_{N k}\left(z_{1}, z_{2}\right) \mathscr{O}_{N k}(x) .
$$

The functions $\Psi_{N k}$ are homogeneous polynomials of degree $N+k$ of the quark coordinates, and, in general, depend on the coupling $a_{*}$. They can be thought of as coordinates of the light-ray operator in the conformal basis spanned by $\mathscr{O}_{N k}$.

Action of the conformal symmetry generators on $\mathscr{O}_{N k}$ follows from (4.1), (4.2) i.e. it is fixed by their transformation properties (scaling dimension and spin). For the light-ray operators, obviously,

$$
\begin{aligned}
i\left[\mathbf{D},\left[\mathscr{O}\left(x ; z_{1}, z_{2}\right)\right]\right] & =\sum_{N k} \Psi_{N k}\left(z_{1}, z_{2}\right) i\left[\mathbf{D}, \mathscr{O}_{N k}(x)\right], \\
i\left[\mathbf{K}^{\mu},\left[\mathscr{O}\left(x ; z_{1}, z_{2}\right)\right]\right] & =\sum_{N k} \Psi_{N k}\left(z_{1}, z_{2}\right) i\left[\mathbf{K}^{\mu}, \mathscr{O}_{N k}(x)\right],
\end{aligned}
$$

and similar for the other generators. Taking into account the expressions in (4.1), (4.2) one obtains after some algebra (recall that the operator $\left[\mathscr{O}\left(x ; z_{1}, z_{2}\right)\right]$ depends implicitly on the auxiliary 
vector $n$ )

$$
\begin{aligned}
i\left[(n \mathbf{P})\left[\mathscr{O}\left(x ; z_{1}, z_{2}\right)\right]\right]= & -S_{-}\left[\mathscr{O}\left(x ; z_{1}, z_{2}\right)\right] \\
i\left[\mathbf{D},\left[\mathscr{O}\left(x ; z_{1}, z_{2}\right)\right]\right]= & \left(\left(x \partial_{x}\right)+2 S_{0}-n \partial_{n}\right)\left[\mathscr{O}\left(x ; z_{1}, z_{2}\right)\right], \\
\frac{i}{2}\left[\mathbf{K}^{\mu},\left[\mathscr{O}\left(x ; z_{1}, z_{2}\right)\right]\right]= & \left(n^{\mu} S_{+}+2 x^{\mu} S_{0}+x^{\mu}\left(x \partial_{x}\right)-\frac{1}{2} x^{2} \partial^{\mu}\right. \\
& \left.+n^{\mu}\left(x \partial_{n}\right)-x^{\mu}\left(n \partial_{n}\right)-(x n) \partial_{n}^{\mu}\right)\left[\mathscr{O}\left(x ; z_{1}, z_{2}\right)\right],
\end{aligned}
$$

where the operators $S_{+}$and $S_{0}$ are defined by their action on the coefficient functions of conformal operators as follows

$$
S_{0} \Psi_{N k}\left(z_{1}, z_{2}\right)=\left(j_{N}+k\right) \Psi_{N k}\left(z_{1}, z_{2}\right), \quad S_{+} \Psi_{N k}\left(z_{1}, z_{2}\right)=(k+1)\left(2 j_{N}+k\right) \Psi_{N k+1}\left(z_{1}, z_{2}\right)
$$

and $j_{N}=\left(\Delta_{N}^{*}+N\right) / 2$ is the conformal spin of the operator. For the special choice $x=0$ in Eq. (4.7) one obtains

$$
i\left[\mathbf{K}^{\mu},\left[\mathscr{O}\left(z_{1}, z_{2}\right)\right]\right]=2 n^{\mu} S_{+}\left[\mathscr{O}\left(z_{1}, z_{2}\right)\right], \quad i\left[\mathbf{D},\left[\mathscr{O}\left(z_{1}, z_{2}\right)\right]\right]=\left(2 S_{0}-\left(n \partial_{n}\right)\right)\left[\mathscr{O}\left(z_{1}, z_{2}\right)\right] .
$$

This definition guarantees that the generators $S_{\alpha}$ satisfy the $S L(2)$ commutation relations.

The expression (3.4) for the generator $S_{0}$ follows directly from the definition (4.8), taking into account that the polynomials $\Psi_{N k}$ are eigenfunctions of the evolution kernel, $\mathbb{H}\left(a_{*}\right) \Psi_{N k}=$ $\gamma_{N}\left(a_{*}\right) \Psi_{N k}$. Next, it follows from Eq. (4.7) that the correlation function of two nonlocal operators defined with respect to different auxiliary vectors, $n$ and $\bar{n},\left[\mathscr{O}_{n}\left(x=0, z_{1}, z_{2}\right)\right]$ and $\left[\mathscr{O}_{\bar{n}}\left(x, w_{1}, w_{2}\right)\right]$, respectively, satisfies the following equation:

$$
\left(2(n \bar{n}) S_{+}^{(z)}-\frac{1}{2} x^{2}\left(\bar{n} \partial_{x}\right)\right)\left\langle\left[\mathscr{O}_{n}\left(z_{1}, z_{2}\right)\right]\left[\mathscr{O}_{\bar{n}}\left(x, w_{1}, w_{2}\right)\right]\right\rangle=0 .
$$

The superscript $S_{+}^{(z)}$ indicates that it is a differential operator acting on $z_{1}, z_{2}$ coordinates and we also assume that $(x \bar{n})=(x n)=0$. The explicit expression for $S_{+}$can be derived from the conformal Ward identity for the corresponding correlator

$$
\left\langle\delta_{+} S_{R}\left[\mathscr{O}^{(n)}\right](z)\left[\mathscr{O}^{(\bar{n})}\right](x, w)\right\rangle=\left\langle\delta_{+}\left[\mathscr{O}^{(n)}\right](z)\left[\mathscr{O}^{(\bar{n})}\right](x, w)\right\rangle+\left\langle\left[\mathscr{O}^{(n)}\right](z) \delta_{+}\left[\mathscr{O}^{(\bar{n})}\right](x, w)\right\rangle,
$$

bringing it to the form (4.10). Here $\delta_{+}$is the transformation generated by the generator $\mathbf{K}_{\bar{n}}=(\bar{n} \mathbf{K})$ and

$$
\delta_{+} S_{R}=4 \varepsilon \int d^{d} x(x \bar{n})\left(\mathscr{L}_{A}+\mathscr{L}_{\xi}+\mathscr{L}_{\text {ghost }}\right)+2(d-2) \bar{n}^{\mu} \int d^{d} x\left(Z_{c}^{2} \bar{c} D_{\mu} c-\frac{1}{\xi} A_{\mu}(\partial A)\right) .
$$

Details of the calculation can be found in Refs. [11, 12]. We stress that considering the correlator of two light-ray operators instead of the Green function of the light-ray operator with quark and antiquark fields considerably simplifies the analysis. Indeed, the Green function is gauge-dependent and does not transform in a proper way under conformal transformations. Another advantage is that the last term in Eq. (4.12) which does not vanish in $d=4$ dimension and explicitly breaks conformal symmetry of QCD Lagrangian, drops out from the correlator of gauge-invariant objects as it is reduced to a BRST variation. 


\section{Two loop kernels}

The two-loop kernel $h^{(2)}(\alpha, \beta)$ contains contributions of two color structures and a term proportional to the QCD beta function,

$$
h^{(2)}(\alpha, \beta)=8 C_{F}^{2} h_{1}^{(2)}(\alpha, \beta)+4 C_{F} C_{A} h_{2}^{(2)}(\alpha, \beta)+4 b_{0} C_{F} h_{3}^{(2)}(\alpha, \beta) .
$$

The noninvariant part of the kernels (5.1) can be restored from the commutator relation Eq. (3.7).

$$
\left[S_{+}^{(0)}, \mathbb{H}^{(2)}\right]=\left[\mathbb{H}^{(1)}, \Delta S_{+}^{(1)}\right] .
$$

Note that $\Delta S_{+}^{(1)}$ (3.5) contains terms in $b_{0}$ and $C_{F}$. Hence the commutator $\left[\Delta S_{+}^{(1)}, \mathbb{H}^{(1)}\right]$ contains two color structures, $b_{0} C_{F}$ and $C_{F}^{2}$, respectively. It follows than that the kernel $h_{2}^{(2)}(\alpha, \beta)(5.1)$ satisfies the homogeneous equation $\left[S_{+}^{(0)}, \mathbb{H}_{2}^{(2)}\right]=0$, alias it is $S L(2)$-invariant and can be written as a function of the conformal ratio, $h_{2}^{(2)}(\alpha, \beta)=h_{2}^{(2)}(\tau)$.

Going through the calculations one gets [12]

$$
\begin{aligned}
h_{1}^{(2)}(\alpha, \beta)= & -\delta_{+}(\tau)\left(\phi_{1}(\alpha)+\phi_{1}(\beta)\right)+\varphi_{1}(\alpha, \beta)+\theta(\bar{\tau})\left[2 \operatorname{Li}_{2}(\tau)+\ln ^{2} \bar{\tau}+\ln \tau-\frac{1+\bar{\tau}}{\tau} \ln \bar{\tau}\right] \\
& +\theta(-\bar{\tau})\left[\ln ^{2}(-\bar{\tau} / \tau)-\frac{2}{\tau} \ln (-\bar{\tau} / \tau)\right]+\left(-6 \zeta(3)+\frac{1}{3} \pi^{2}+\frac{21}{8}\right) \delta(\alpha) \delta(\beta), \\
h_{2}^{(2)}(\alpha, \beta)= & \frac{1}{3}\left(\pi^{2}-4\right) \delta_{+}(\tau)-2 \theta(\bar{\tau})\left[\operatorname{Li}_{2}(\tau)-\operatorname{Li}_{2}(1)+\frac{1}{2} \ln ^{2} \bar{\tau}-\frac{1}{\tau} \ln \bar{\tau}+\frac{5}{3}\right] \\
& -\theta(-\bar{\tau})\left[\ln ^{2}(-\bar{\tau} / \tau)-\frac{2}{\tau} \ln (-\bar{\tau} / \tau)\right]+\left(6 \zeta(3)-\frac{2}{3} \pi^{2}+\frac{13}{6}\right) \delta(\alpha) \delta(\beta), \\
h_{3}^{(2)}(\alpha, \beta)= & -\delta_{+}(\tau)\left[\ln \bar{\alpha}+\ln \bar{\beta}+\frac{5}{3}\right]-\theta(\bar{\tau})\left[\ln (1-\alpha-\beta)+\frac{11}{3}\right]+\frac{13}{12} \delta(\alpha) \delta(\beta),
\end{aligned}
$$

where $\bar{\tau}=1-\tau$, and the functions $\phi_{1}(\alpha)$ and $\varphi_{1}(\alpha, \beta)$ are given by the following expressions

$$
\begin{aligned}
\phi_{1}(\alpha)= & -\ln \bar{\alpha}\left[\frac{3}{2}-\ln \bar{\alpha}+\frac{1+\bar{\alpha}}{\bar{\alpha}} \ln \alpha\right] \\
\varphi_{1}(\alpha, \beta)= & -\theta(1-\tau)\left[\frac{1}{2} \ln ^{2}(1-\alpha-\beta)+\frac{1}{2} \ln ^{2} \bar{\alpha}+\frac{1}{2} \ln ^{2} \bar{\beta}-\ln \alpha \ln \bar{\alpha}-\ln \beta \ln \bar{\beta}\right. \\
& \left.-\frac{1}{2} \ln \alpha-\frac{1}{2} \ln \beta+\frac{\bar{\alpha}}{\alpha} \ln \bar{\alpha}+\frac{\bar{\beta}}{\beta} \ln \bar{\beta}\right] .
\end{aligned}
$$

\section{Conclusion}

Our result for the two-loop evolution kernels of flavor-nonsinglet operators in Eqs. (5.1), (5.3) is equivalent to the corresponding evolution equation for GPDs obtained in Ref. [3] in momentum space and has manifest $S L(2)$-symmetry properties. This feature presents the crucial advantage of the light-ray operator formalism which makes this technique attractive for higher-order calculations. Exact conformal symmetry of QCD at the critical point is very helpful on intermediate steps of the calculation as it provides one with algebraic group-theory methods to calculate the commutators of integral operators that appear in Eqs. (3.7). Evolution equations for GPDs can be obtained from our expressions by a Fourier transformation which is rather straightforward, cf. [22]. 


\section{References}

[1] A. V. Belitsky and D. Müller, Nucl. Phys. B 527 (1998) 207.

[2] A. V. Belitsky and D. Müller, Nucl. Phys. B 537 (1999) 397.

[3] A. V. Belitsky, A. Freund and D. Müller, Nucl. Phys. B 574 (2000) 347.

[4] S. Moch, J. A. M. Vermaseren and A. Vogt, Nucl. Phys. B 688 (2004) 101.

[5] A. Vogt, S. Moch and J. A. M. Vermaseren, Nucl. Phys. B 691 (2004) 129.

[6] Y. .M. Makeenko, Sov. J. Nucl. Phys. 33 (1981) 440 [Yad. Fiz. 33 (1981) 842].

[7] D. Müller, Z. Phys. C 49 (1991) 293.

[8] D. Müller, Phys. Rev. D 49 (1994) 2525.

[9] D. Müller, Phys. Rev. D 58 (1998) 054005.

[10] A. V. Belitsky and D. Müller, Phys. Lett. B 417 (1998) 129.

[11] V. M. Braun and A. N. Manashov, Eur. Phys. J. C 73 (2013) 2544

[12] V. M. Braun and A. N. Manashov, Phys. Lett. B 734 (2014) 137.

[13] V. M. Braun, G. P. Korchemsky and D. Mueller, Prog. Part. Nucl. Phys. 51 (2003) 311.

[14] I. I. Balitsky and V. M. Braun, Nucl. Phys. B 311 (1989) 541.

[15] V. M. Braun, S. E. Derkachov, G. P. Korchemsky and A. N. Manashov, Nucl. Phys. B 553 (1999) 355.

[16] T. Banks and A. Zaks, Nucl. Phys. B 196 (1982) 189.

[17] A. Hasenfratz and P. Hasenfratz, Phys. Lett. B 297 (1992) 166.

[18] J. A. Gracey, Phys. Lett. B 373 (1996) 178.

[19] J. A. Gracey, Phys. Lett. B 322 (1994) 141.

[20] J. F. Bennett and J. A. Gracey, Nucl. Phys. B 517 (1998) 241.

[21] M. Ciuchini, S. E. Derkachov, J. A. Gracey and A. N. Manashov, Phys. Lett. B 458 (1999) 117;

[22] V. M. Braun, A. N. Manashov and B. Pirnay, Phys. Rev. D 80 (2009) 114002 [Erratum-ibid. D 86 (2012) 119902]. 